\title{
Zeichen lesen und Zeichen deuten
}

\author{
Zur Geschichte der Medizinischen Semiotik
}

von Antoinette Stettler

\section{Einleitung: Zeichen lesen und Zeichen deuten}

«Der Arzt wird den Namen keiner Krankheit vermissen, die in diesem Buch nicht ausdrücklich genannt ist. Denn alle ... wird er an denselben Zeichen erkennen.»

(Hippokrates, Prognostikon, Schluß)

«Man hat in neuerer Zeit auch die Zeichen durch das Gehör, mit Hülfe des Stethoskopes oder der Perkussion, zur Diagnose bei Brustkrankheiten sehr empfohlen. Aber diese Zeichen sind sehr trüglich ..., wenn man nicht die anderen Zeichen zu Hülfe nimmt, die allein schon zur Diagnose hinreichen.»

(C. W. Hufeland, Enchiridium medicum, Berlin 1857, 1. Aufl. 1836, S. 112)

Diese beiden Zitate liegen rund 2200 Jahre auseinander. Sie betreffen aber denselben Sachverhalt des ärztlichen Alltags, indem sie das Problem der richtigen Diagnose der Krankheit stellen. Während Hippokrates aus den Zeichen den Namen der Krankheit liest, warnt Hufeland skeptisch vor trügerischen Zeichen, die nichts oder etwas Falsches bedeuten. Diagnose wäre demnach Zeichenlesung und Zeichendeutung.

Beides, Zeichenlesung und Zeichendeutung, sind im französischen und angelsächsischen Sprachbereich als medizinische Semiotik noch heute propädeutisches Lehrfach. Aus den Lehrplänen deutschsprachiger Universitäten verschwand indessen die medizinische Semiotik vor rund 150 Jahren.

Seit dem Ende des 19. Jahrhunderts wird Semiotik außerdem als Wissenschaft betrieben, die sich in der Nachfolge von Ferdinand de Saussure (1857-1913) vor allem der Sprache, aber auch der Lesung anderer Zeichensysteme annimmt. Mit Charles Peirce (1839-1914) und Charles Morris (1938) erhielt die Semiotik eine moderne philosophische Grundlage, die aus Sprachwissenschaft, Kunstinterpretation und vor allem auch aus der Informatik kaum mehr wegzudenken ist. 
Semiotik und Medizin haben anscheinend eine so enge Verbindung, daß Thomas Seboek sogar so weit ging, den Hippokrates als den eigentlichen Begründer der Semiotik zu bezeichnen ${ }^{1}$.

Hippokrates verdient den Ruhm des Urvaters der Semiotik insofern, als er die medizinische Zeichenlesung zu systematisieren begann. Doch ist das Verhältnis der allgemeinen Semiotik zur medizinischen Semiotik noch nicht hinlänglich genug untersucht, als daß heute schon behauptet werden könnte, Hippokrates habe generell die Semiotik erfunden. Deshalb soll im folgenden ein Beitrag zur Frage nach der Art des Verhältnisses der Medizin zur Semiotik im Laufe der Zeit geleistet werden.

$\mathrm{Zu}$ diesem Zweck muß zuerst umschrieben werden, was unter medizinischer Semiotik verstanden werden soll. In allgemeinster Form ausgedrückt, heißt Semiotik Zeichenlehre (von «semeion», griech., das Zeichen). Wie an anderer Stelle nachgewiesen wurde ${ }^{2}$, beruht die medizinische Diagnose tatsächlich auf Zeichenprozessen, sogenannten Semiosen, verschiedener Stufen und verschiedener Abstraktionsgrade. Darauf soll kurz eingegangen werden.

Will man das ärztliche Handeln, das dank Ausbildung und Erfahrung weitgehend ohne weitere Reflexion geschieht, in seine Abfolgen aufgliedern, so ergibt sich aller Wahrscheinlichkeit nach folgender Sachverhalt. Der Arzt wird zuerst die verschiedenen anamnestischen Angaben, die Symptome, die Labor- und Röntgenbefunde als je einzelne Zeichen zusammen gruppieren und diese zu einem Krankheitsbild ordnen, dem er einen Namen gibt. Die Zeichen Z 1, Z 2, Z 3, usw. ergeben demnach den Namen der Krankheit oder des Syndroms. Dabei untersucht der Arzt das Verhältnis der Zeichen untereinander. Er bewegt sich in der syntaktischen Dimension der Semiose. Die syntaktische Dimension behandelt die Relation der Zeichen zueinander.

Ein Arzt, welcher dergestalt den Namen der Krankheit «gelesen» hat, geht aber noch einen Schritt weiter. Der durch Zeichenlesung erschlossene Name hat für ihn eine Bedeutung, indem er ihn nun in der Prognose, in der Pathologie, im sozialen Umfeld usw. einordnen kann. Mit der Festlegung der Bedeutung der Krankheit begibt sich der Arzt in die semantische Dimension der Semiose. Er ist von der Zeichenlesung zur Zeichendeutung gekommen.

Schließlich wird der Arzt eine Handlung vornehmen oder veranlassen. Damit befindet er sich auf der pragmatischen Ebene der Semiose.

Zur Veranschaulichung der drei Dimensionen der Semiose diene das folgende Dreieckschema: 
Schema der ärztlichen Semiose (Zeichenprozeß)

$\mathrm{Z} 1 \ldots \mathrm{Z}_{\mathrm{n}}$ : Anamnese, Symptome, Befunde = Name der Krankheit: Syntaktik

Bedeutung der Zeichen in bezug auf Prognose, Pathologie, soziales Umfeld, usw.:
Semantik

Therapie im weitesten Sinne: Pragmatik

Solch ein Dreieckschema gilt auch für Semiosen in anderen Bereichen der Wissenschaft und wird als generell gültig für den semiotischen Prozeß angesehen. Syntaktische Zeichenlesung, semantische Zeichendeutung sowie daraus folgendes Handeln gelten universell, wie das von der allgemeinen Semiotik postuliert wird ${ }^{3}$.

Wenn nun in der folgenden Arbeit untersucht wird, wie sich die medizinische Semiose historisch dargestellt hat, sollen vor allem die syntaktische und die semantische Dimension der Semiose betrachtet werden, d.h. welche Zeichen überhaupt gelesen und wie sie gedeutet wurden.

\section{Die Syntaktik in der Zeichenlehre der Hippokratischen Medizin und die hippokratische Tradition}

\subsection{Zum Corpus Hippocraticum}

Im «Prognosticon», einer als authentisch angesehenen Schrift, gibt Hippokrates eine Anleitung für das praktische ärztliche Vorgehen.

«Der Arzt braucht den Namen keiner Krankheit zu vermissen. Er wird sie alle an denselben Zeichen erkennen ${ }^{4}$.

Um die Krankheiten zu erkennen und zu ihren Namen zu ordnen, soll der Arzt auf folgende Zeichen (semeia) achten:

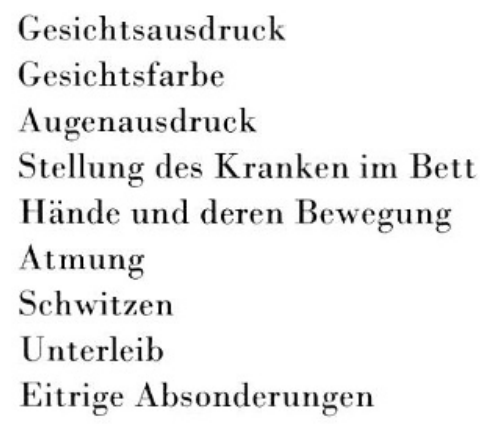

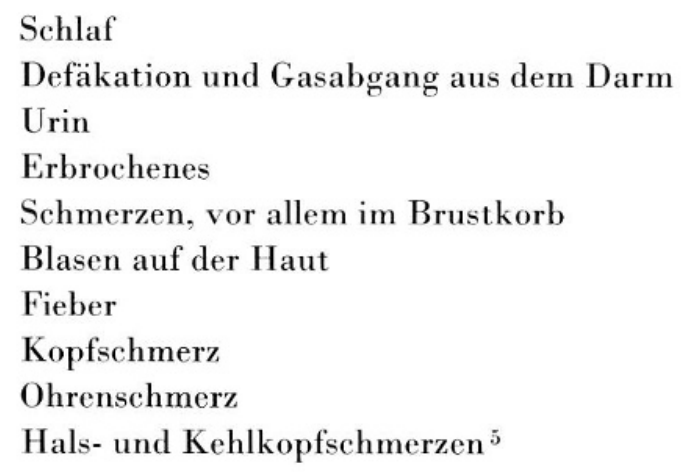


Diese hier in einer Liste wiedergegebenen Arten menschlicher Befindlichkeit können so oder anders vom Gewohnten abweichen. In der Abweichung zeigt sich der Krankheitszustand. Man nennt dieses Vorgehen in einer Schablone gemeinhin den «hippokratischen Empirismus» und feiert Hipporates dafür, daß er überhaupt beobachtet habe. Die hippokratischen Schriften mit Empirismus zu charakterisieren, ist indessen nicht genug. Denn dort wurde noch ein Zusätzliches getan: Aus den verschiedenen Zeichen fügte man Krankheitsbilder, d.h. die Namen der Krankheiten, zusammen. Das ist Arbeit in der syntaktischen Dimension der Semiose. Wir illustrieren das Vorgehen des hippokratischen Arztes mit zwei Beispielen. Das erste zeigt die Aufstellung eines individuellen Krankheitsbildes, das zweite weist als Titel den Namen der Krankheit auf:

Philiskos wohnte an der Stadtmauer. Er wurde bettlägerig. Am ersten Tage hohes Fieber. Schweißausbruch. Schlimme Nacht. Am zweiten in jeder Hinsicht starke Verschlimmerung. Abends spät infolge eines Klistiers normaler Stuhlgang. Ruhige Nacht. Am dritten morgens und zum Mittag schien er fieberfrei; aber gegen Abend hohes Fieber mit Schweiß. Durstig. Zunge ausgetrocknet, schwärzlicher Urin. Die Nacht schlecht, schlaflos. Alle möglichen Wahnvorstellungen. Am vierten allgemeine starke Verschlimmerung. Urin schwarz. Bessere Nacht. Urin sah besser aus. Am fünften gegen Mittag leichtes Nasenbluten, tiefrot. Urin mehrfarbig, enthielt darin schwebende, runde, samenähnliche, zusammenhanglose Gebilde, die sich nicht setzten. Nach Einführung eines Zäpfchens ging ganz wenig unter Winden ab. Die Nacht schlecht, wenig Schlaf. Redete viel, meist dummes Zeug. Hände und Füße ganz kalt, nicht mehr warm zu kriegen. Urin schwarz, schlief etwas gegen Morgen. Verlust der Sprache. Kalter Schweiß, Extremitäten bläulich. Am sechsten gegen Mittag starb er. Sein Atem ging bis zuletzt in großen Intervallen, tief heraufholend, als ob er ihn zurückrufen wollte. Die Milz schwoll zu einer runden Wölbung an. Kalter Schweiß bis zuletzt. Die schweren Anfälle an geraden Tagen ${ }^{6}$.

«Weißer Fluß: fließt weiß wie Harn eines Esels. Schwellungen im Gesicht. Auch die Partien unter beiden Augen schwellen an. Die Augen selbst sind wässerig und nicht ganz klar; es fehlt der Glanz. Die Kranke ist triefäugig und blickt stumpf. Thre Haut ist sardellenfarben und voller kleiner Bläschen. Der Unterleib schwillt an. Auf den Wangen entsteht allmählich ein rötlicher, feiner, wässeriger, übler Ausschlag. Schwellungen an den Beinen, und wenn man mit dem Finger darauf drückt, entsteht ein Eindruck wie beim Brotteig. Der Mund füllt sich mit Speichel. Magenschmerzen, wenn sie noch nüchtern sind. Erbrechen einer scharfen wässerigen Flüssigkeit. Und wenn sie bergan gehen, fangen sie alsbald an zu keuchen. Atemnot tritt ein. Die Beine werden eiskalt; die Patientin hat keine Gewalt mehr über ihre Knie, und im Munde macht sich ein böser Ausschlag bemerkbar. Ihre Oberschenkel durchzucken Schmerzen. Die ganzen unteren Partien des Körpers werden kalt, vom Unterleib beginnend bis zu den Füßen. Die Fußsohlen sind eingeschlafen; sie können nicht auftreten ... Frauen in solchem Zustand kann man schwer von ihrem Leiden heilen. Denn ihr Alter ist vorgerückt und ihr Gebrest altert mit ihnen, falls nicht von selbst irgendein glücklicher Umstand eintritt und sie befreit.»? 
Selbstverständlich gehen die hippokratischen Ärzte empirisch vor, wie immer wieder gesagt wird, aber sie erweisen sich eben auch immer als syntaktische Semiotiker, indem sie die Vielfalt der Zeichen zu Krankheitsbildern gruppieren.

Die Krankheitsnamen, welche mit Hilfe des Corpus Hippocraticum zusammengefügt werden können, haben nicht mehr viel mit unseren heutigen Krankheitsnamen zu tun. Solche Fragen nach der retrospektiven Diagnose beschäftigen bekanntlich einen Teil der Medizingeschichte. Ihre Lösung kann nicht einfach in der Übertragung heutiger Krankheitsnamen auf die historisch gegebenen bestehen. Wenn wir dennoch jetzt diesen Versuch anstellen, bietet sich für den Philiskos derjenige der Sepsis an, für die Frauen der der Ödeme. «Sepsis» und «Ödem» sind aber im 20. Jahrhundert keine Namen von Krankheiten mehr; sie sind vielmehr zu Zeichen geworden, die auf Krankheiten hindeuten, die völlig andere Namen haben. Das tut indessen der syntaktischen Arbeit der hippokratischen Ärzte keinen Abbruch. Denn sie hatten sich mit ihrer Zeichenlesung den Krankheitsfällen zugewandt, die sich ihnen am häufigsten präsentierten. Das waren relativ unspezifische Marasmus- und Endzustände von Krankheiten, die heute dank veränderten Zielvorstellungen und Erkenntniszuwachs sehr oft schon in ihren Anfängen bekämpft oder verzögert werden können.

Wir sind es heutzutage gewohnt, daß zahlreiche Krankheiten den Namen ihres Erstbeschreibers tragen. Es wirkt nun beinahe tragisch, daß man heute des Arztes Hippokrates, dem wir die ersten semiotisch/syntaktisch erstellten Krankheitsbilder verdanken, nur noch mit dem Ausdruck der «facies hippocratica» gedenkt, wenn man von der Schulter- und Wirbeleinrenkung nach der hippokratischen Methode absieht. Die «facies hippocratica» beschreibt das unmittelbare Bevorstehen des Todes. Der erste Arzt, der in der Geschichte mit so lebendigen Krankheitsbeschreibungen an den Tag tritt, erinnert heute mit seinem Eigennamen an die tödliche Krankheit.

Abschließend ist noch kurz darauf einzugehen, daß die hippokratischen Ärzte die syntaktische Zeichenlesung auch nicht ausschließlich um ihrer selbst willen betrieben. Worauf der Name der Krankheit hindeutete, ist mit einem Zitat, das zu Anfang des «Prognosticon»steht, zu verdeutlichen:

«Der beste Arzt scheint mir der zu sein, der sich auf Voraussicht versteht. Denn wenn er den gegenwärtigen und den ihm vorangegangenen und den zukünftigen Stand einer Krankheit schon vorher erkennt und den Kranken vorhersagt, ... werden sie ihm vertrauen.» 
Von der semantischen Dimension der Semiose her betrachtet, bedeutet in der hippokratischen Medizin der Name der Krankheit die Prognose. Aus dem Blickwinkel der Semiotik bestätigt sich demnach, was anhand anderer Kriterien als das prognostische Element der hippokratischen Medizin beschrieben worden ist ${ }^{8,9}$.

\subsection{Die Weiterentwicklung der syntaktischen Dimension der Zeichenlehre}

Von der Antike bis ungefähr 1850 wurde Hippokrates nicht kontinuierlich, aber immer wieder als Autorität angerufen, die für das aktuelle medizinische Verständnis ausschlaggebend war.

Es sollen im Folgenden diejenigen Momente syntaktischer Anwendung der hippokratischen Zeichenlehre dargestellt werden, die auch heute noch Gültigkeit haben. Darunter verstehen wir, wie dargelegt, die Zeichenlesung, d. h. aus der Addition von Zeichen plus Zeichen entsteht ein Krankheitsbild. Daraus wird der Name der Krankheit abgelesen.

Ohne den Anspruch auf Vollständigkeit zu erheben, sei für eine syntaktische Leistung medizinischer Art zunächst Aretaios von Kappadokien genannt, der für die Diphterie und die Lepra arabum die erste Beschreibung gab $^{10}$. Ferner ist der arabische Arzt Rhazes zu erwähnen, dem es gelang, durch Zeichenlesung die Pocken und die Masern zu identifizieren und differenzieren ${ }^{11}$. Es wäre die zahlreichen Ärzte aufzuzählen, welche im Gefolge des «Schwarzen Todes» von 1347 auch für uns verständliche Beschreibungen der Bubonenpest verfaßten ${ }^{12}$. Schließlich ist noch auf Thomas Sydenham hinzuweisen, der sich unter ausdrücklicher Nachahmung der hippokratisch/syntaktischen Methode durch die abgrenzende Darstellung der Krankheit Hysterie/Hypochondrie auszeichnete ${ }^{13}$.

Die Medizingeschichte legte bisher vor allem den Akzent darauf, daß solche Erstbeschreiber imstande gewesen waren, diese in der Realität tatsächlich existierenden Krankheitsbilder zu erkennen. Ohne ihren Verdiensten Abbruch zu tun, sei zu bedenken gegeben, daß jeder in seiner Zeit den Vorzug der großen Zahl ähnlicher Krankheitsfälle genoß. Kamen ihm in der hippokratischen Zeit vorwiegend Septikämien zu Gesicht, waren es für andere Zeiten die genannnten Epidemien. Mit dem 16. und 17. Jahrhundert fingen die Ärzte an, Krankenjournale zu führen, die ihnen vergleichbare Fälle lieferten ${ }^{14}$. Im 18. und 19. Jahrhundert zogen die Ärzte aus den dann gegründeten Spitälern die Vielfalt vergleichbarer Krankheitsfälle. 
Es ist schon geschätzt worden, daß wir der syntaktischen Methode zufolge, heutzutage an die 30000 Krankheitsbilder und damit Krankheitsnamen kennen. Diese Zahl dürfte zu hoch greifen. Denn man muß davon ausgehen, daß es zu allen Zeiten auch «fiktive» Krankheitseinheiten gegeben hatte.

\section{Die Semantik in der Zeichenlehre des Galen und in der galenischen Tradition}

\subsection{Zu den galenischen Büchern über Zeichenlehre und Pathologie}

Zur Bestimmung der galenischen Zeichenlehre und Pathologie berufen wir uns auf die Bücher «De morborum differentiis et causis», «De symptomatum differentiis et causis», «De pulsibus», «De urinis», «De locis affectis» sowie «Introductio seu medicus». Sie enthalten diejenigen Lehren, die für das Mittelalter und die Renaissance entscheidend wurden. Diese um 150 n. Chr. entstandenen Bücher führen das Wort «semeion» überhaupt nicht im Titel, sind aber dennoch als Zusammenstellung der spätantiken Zeichenlehre anzusehen.

Genauso wie die hippokratischen Ärzte weist Galen zunächst darauf hin, auf welche Zeichen der Arzt zu achten habe, ja er widmet mit «De locis affectis» der erkrankten Körperstelle, sei es nun ein Tumor, ein Geschwür, ein Furunkel usw., ein eigenes Werk.

Des ferneren führt Galen neben dem «semeion» einen neuen Begriff ein, den er «symptoma» nennt. Auch darauf müsse der Arzt sein Augenmerk richten. Dieses Wort heißt in seiner ursprünglichen Bedeutung nichts weiter als Zufall, Unfall, Unglück, demnach das, was dem Menschen, von einer göttlichen Macht gesendet, zustößt. Dadurch entsteht ein Bedeutungsumfeld, das sich einerseits mit dem vom Kranken empfundenen Begriff der «Krankheit» überschneidet, anderererseits sich aber auch mit dem Begriff deckt, den sich der Arzt vom Zeichen bildet. «Symptoma» (lat. «accidens») ist demnach ein doppeldeutiger Begriff, der das subjektiv empfundene und das objektiv feststellbare Element enthält.

Da Galen für Ärzte schrieb und sich für den Aspekt des subjektiven Unglücks weniger interessierte, galt für ihn die objektive Bedeutung des Symptombegriffs. Er unterschied zwischen «semeion» und «symptoma» folgendermaßen: 
Symptom ist der krankhafte Befall, welcher der Krankheit wie der Schatten dem Körper folgt und sich auswirkt in der gestörten Funktionsweise, der verdorbenen Qualität der Säfte sowie in der Auswirkung von beidem, nämlich in den veränderten Körperausscheidungen. Diese letzteren sind die Zeichen, semeia, und geben Hinweis auf dergestalt gestörte Funktion oder Säfte. Die Zeichen sind pathognomonisch ${ }^{15}$ in allererster Linie. Erst in zweiter Linie sind die Zeichen prognostisch, wie man das in der hippokratischen Tradition gesehen hat.

Symptome und Zeichen, beide sind pathognomonisch und deuten demgemäß auf die Lehre von der Krankheit, auf die "pathologia», eine Wortprägung, die übrigens auch aus den galenischen Schriften stammt.

Der galenische Symptombegriff schließt schon die vollausgebildete sogenannte Vier-Säfte-Pathologie mit ein. Es ist zwar nicht abzustreiten, daß auch im Corpus Hippocraticum gewisse Zeichen auf eine solche Pathologie hin gedeutet werden. Doch muß diese rekonstruiert werden, weil sie meistenfalls nicht ausdrücklich erwähnt ist. Diese so erschlossene hippokratische Pathologie beruht bekanntlich auf der Lehre von den vier Temperamenten, die - gestört - die Krankheit verursachen.

Weil Galen das Symptom mit «functio laesa» und «qualitas vitiata» definiert, m.a.W. weil nun das Symptom auf die «pathologia» hindeutet, wird notwendigerweise das Symptom das wichtigere Kriterium für den Arzt, als es das Zeichen/semeion je gewesen war. An dieser Bevorzugung des Symptoms gegenüber dem Zeichen zeigt sich Galens Vorgehen in der semantischen Dimension der Semiose. Die Bedeutung der Zeichen und der Symptome in Beziehung auf die Pathologie, nicht die Zeichenlesung als solche, gewinnt an Wichtigkeit. Die galenischen Symptome sind recht eigentlich semantisch.

Die Zeichenlehre, so wie sie von Hippokrates verstanden wurde als die für den Arzt mit seinen Sinnen wahrnehmbaren Bestandteile des Namens der Krankheit, wird zwar durch Galen mitnichten vergessen. Aber sie wird in den Rang einer Hilfswissenschaft verwiesen, die der Pathologie untergeordnet zu sein hat. "Semeiotica», wie sie (Pseudo-)Galen nennt ${ }^{16}$, wird zum Grundunterricht der vorbereitenden Medizin. Im Vordergrund steht bei Galen allemal die Lehre von der Krankheit als «functio laesa» und «qualitas vitiata». Denn nur das Symptom macht das Zeichen, das dann am Puls, Urin usw. abgelesen werden kann. 


\subsection{Die Weiterentwicklung der semantischen Dimension der Zeichenlehre}

Avicenna (arab.Ibn-Sina, 928-1037) schrieb den «Canon medicinae», der die abendländische Medizin bis zur Renaissance ausschlaggebend mitgestaltete. Uns interessiert der erste Abschnitt des «Canon», in welchem die Pathologie, mithin die Semiotik, zur Sprache kommt ${ }^{17}$.

Wohl bezieht sich die Symptomen- und Zeichenlehre auf Galen, aber Avicenna sagt:

«Symptom heißt Zufall, Unfall in betreff auf sich selbst und im Zusammenhang mit dem (Tatbestand), mit welchem es auftritt, heißt aber Bedeutung für den Arzt, der sich davon leiten läßt. » ${ }^{18}$

Ferner kann das Symptom gleichzeitig auch Krankheit und Krankheitsursache sein. Es kommt nur auf das zeitliche Verhältnis an. Beispielsweise ist Fieber bei Phthise das Symptom des Ulcus in der Lunge. Wenn Fieber Magenschwäche macht, ist es Ursache; an sich selbst ist Fieber aber eine Krankheit ${ }^{18}$.

Wir stellen bei Avicenna eine Vereinfachung des Symptombegriffes fest. Symptom, auch wenn subjektiv und vieldeutig im Sinne von Hinweis auf Ursache oder Krankheit, ist für den zugezogenen Arzt eindeutig als Zeichen aufzufassen; die galenische Zweiteilung von «symptoma» und «semeion» ist somit aufgehoben. Was Avicenna allerdings mit Galen verbindet, ist der Tatbestand, daß Symptom und Zeichen auf die Vier-Säfte-Pathologie hindeuten.

So stellte sich die Lage in den Jahren vor Beginn des Buchdruckes dar. Die Ärzteschaft stützte sich auf Avicenna, entdeckte aber etwa gleichzeitig die ursprünglichen Werke des Galen, hatte demnach aus zwei - wenn auch bis zu einem gewissen Grade einheitlichen - Quellen zu schöpfen.

Im Jahre 1554 publizierte Jean Fernel sein grundlegendes Werk «Pathologia». Ein Kapitel darin ist mit «De symptomatibus et signis» übertitelt und darf als Summe aus Avicennas und Galens Zeichenlehre gelten. Nebenbei sei bemerkt, daß sich um 1550 für «symptoma/accidens» der griechische Ausdruck Symptom, für «semeion/signum» aber der lateinische Ausdruck durchzusetzen scheint. Fernel ist apodiktisch:

«Omne symptoma signum est, non omne signum symptoma.» ${ }^{19}$

(«Jedes Unglück oder Schicksalsschlag ist zwar ein Zeichen, aber nicht jedes Zeichen ist Unglück.»)

Weiter definiert Fernel: 
«Symptoma conspicuus est, foras sedet» ${ }^{20}$. («Das Symptom ist einsehbar und sitzt außen [am Körper].») Das «signum» hingegen, aus Puls, Urin und Körperausscheidungen aller Art gelesen, weist auf innere Vorgänge.»

Fernels Anliegen war nicht in erster Linie die Semiotik, sondern die Pathologie. Dennoch zeigt die Einbettung des Kapitels «De symptomatibus et signis» in die «Pathologia», daß Zeichenlesen und Zeichendeuten, ob innen oder aussen, eindeutig der Pathologie untergeordnet waren. Die semantische Tradition, die mit Galen ihren Anfang nahm, erreichte mit Fernel einen Höhepunkt. Semantische Zeichendeutung war Teildisziplin der Krankheitslehre.

Fernels «Pathologia» wurde noch 200 Jahre nach ihrem Erscheinen als Lehrbuch gebraucht. Auch im «Dictionnaire des Sciences Médicales» (1812-1822) ist seine Symptomdefinition erwähnt ${ }^{21}$. Man darf vermuten, daß die heutzutage im französischen und angelsächsischen Sprachbereich gebräuchliche Unterscheidung zwischen subjektivem Symptom und objektivem Signum auf den Dictionnaire und damit auf Fernel zurückgeht.

Fernels «Pathologia» fällt in eine Umbruchszeit der Medizin. Nicht nur war damals der «alte» Avicenna und der «ursprüngliche» Galen bekannt. Fernel war vielmehr auch Zeitgenosse von Andreas Vesal und Antonio Benivieni. Der erstere schuf bekanntlich 1543 mit der «Fabrica» die erste ausführliche Anatomie des Menschen, der letztere versuchte erstmals auch innere Orte der Krankheit in einen Zusammenhang mit den Krankheitszeichen zu bringen ${ }^{22}$. Er wird daher als einer der Begründer der anatomischen Pathologie angesehen. Es ist auch eine Zeit des Umbruches insofern, in welcher die semantische Dimension mit ihren auf die Vier-Säfte-Pathologie bezogenen Krankheitszeichen einer mehr auf die syntaktische Dimension ausgerichteten Zeichenlesung Platz machte.

\section{Der Neubeginn der Semiotik: Zeichenlesung als traditionelle und empirische Disziplin}

\subsection{Das Buch «Semeiotica» von Jacques Aubert (Jacobus Albertus)}

In den letzten Jahrzehnten des 16. Jahrhunderts, als der Buchdruck immer populärer wird, beginnt die Zeit der medizinischen Monographien. Nicht mehr nur «Summa» oder «Canon», d.h. umfassende Werke über die Heilkunde, sondern auch Einzelgebiete werden herausgenommen, bearbeitet und an den Tag gebracht. Eines der ersten, wenn nicht das erste der 
Bücher mit Titel «Semeiotica» stammt vom französischen Arzt Jacques Aubert, der 1586 in Lausanne verstarb. Sein Buch wurde verschiedene Male verlegt. Man besitzt Ausgaben von Lausanne 1587, Lyon (oder Genf?) 1596 und Basel 1634.

Wir gehen davon aus, daß um 1580 Semiotik nicht mehr nur syntaktische Zeichenlesung im eingangs dargestellten Sinne des Hippokrates sein konnte. Dafür sind zu viele Jahrhunderte der Zeichendeutung auf die Vier-SäftePathologie hin an der Medizin vorbeigegangen. Um so interessanter scheint es nun, einen Blick darauf zu werfen, was eine der ersten Monographien über Semiotik zu diesem Gebiet ärztlichen Handelns zu sagen hat.

Das Buch «Semeiotica» ${ }^{23}$, das rund 60 Seiten umfaßt, hat 20 Kapitel recht unterschiedlicher Länge. Im Vorwort beruft sich Aubert auf Galens «De locis affectis», das uns als wichtig für die Syntaktik der Zeichenlehre begegnet ist. Der Autor will vom Arzt folgende Zustände beachtet wissen:

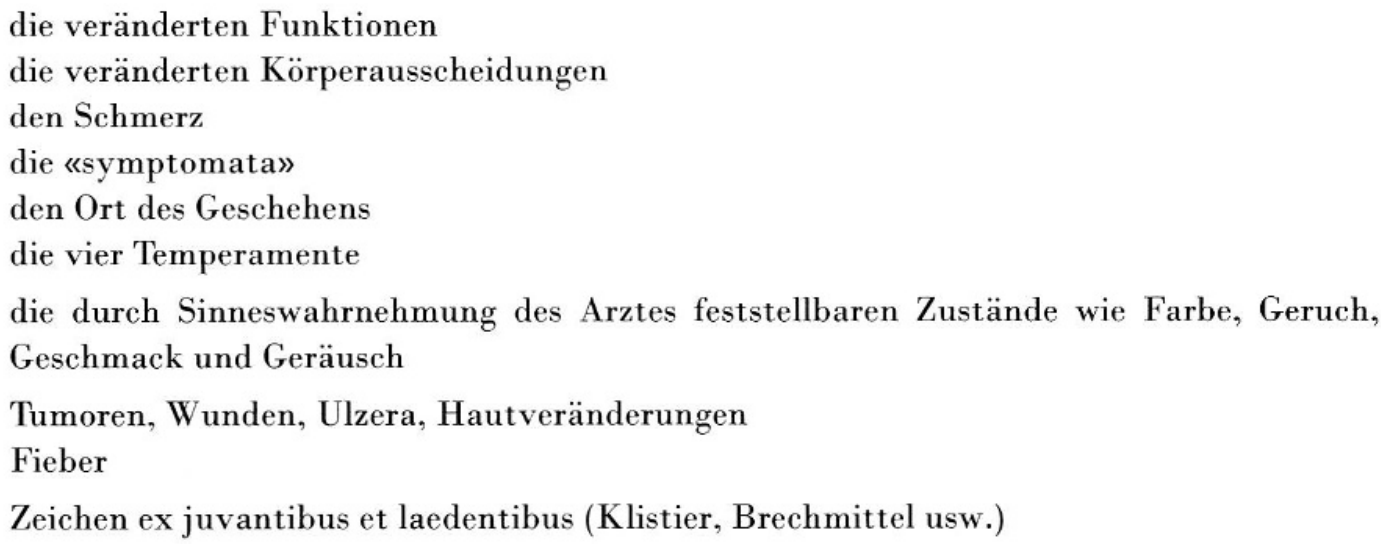

Der heutige Leser ist geneigt, dies alles als ein ziemlich komplettes Inventar ärztlicher Beobachtung anzusehen. Für Tumoren, Ulzera, Hautefflorreszenen sowie für die Schmerzzustände ist bei Aubert schon das Vokabular vorgegeben, das wir zum großen Teil auch heute noch benützen.

Verglichen mit der Theoriediskussion um 1550, die z. B. bei Fernel um das Thema der Definition von Symptom und Zeichen kreiste, wirkt Aubert mehr praktisch. Er betont, was der Arzt «sensibus suis» über die Krankheit erfahren kann. Verglichen andererseits mit der beinahe lapidaren Liste des «Prognostikon», fällt bei Aubert allerdings die Neigung zur Abstraktion, aus welcher mittelbar die Zeichen gelesen werden sollen. Als Beispiel diene der Schmerz. War es bei Hippokrates noch der Ohrschmerz, das Halsweh usw., sind es bei Aubert differenzierte Schmerzzustände an sich, auf die geachtet werden muß (akut, stechend, bohrend, kolikartig usw.). 
Eine weitere Abstraktion ist die Körperhitze. Stand bei Hippokrates die Körperhitze des Philiskos zur Diskussion, schließt sich bei Aubert die ganze ausgeklügelte Fieberlehre, die wir schon bei Avicenna finden, mit an. Schließlich sind alle Körperausscheidungen unter einer einzigen Abstraktion zusammengefaßt, die Urinfarbe an sich, die Niederschläge an sich, usw.

Worauf deuten nun diese Zeichen hin? Aubert ist sehr wortreich: seine «signa» indizieren (indicant), denotieren (denotant), bedeuten (significant), erlauben die Beurteilung (iudicant), ermöglichen die Diskussion (arguunt), geben Hinswei (innotescant). Sie geben Hinweis selbstverständlich auf die damals akzeptierte Vier-Säfte-Pathologie, aber doch in ganz entscheidendem Ausmaß auch auf den «locus affectus», den Ort des Krankheitsgeschehens. Besonders die Kapitel über innere Tumoren, Wunden und Ulzera scheinen als ein Echo auf die besser bekannt werdende anatomische Pathologie seiner Zeit.

Aus dieser Neigung zur Abstraktion geht hervor, daß der Zeichenträger, das «semeion» des Hippokrates, nun nicht mehr das ärztliche Sensorium als Ganzheit betrifft. Es ist nicht mehr das unmittelbare Sinnenerlebnis, das der Arzt hat, wenn er zu einem Kranken tritt. Das Sinneserlebnis ist vielmehr filtriert durch die Abstraktionen der erwähnten Art.

Dadurch wird der Zeichenträger nunmehr zu einer Mischung aus je nach Arzt ungleichen Teilen unmittelbarer Sinneserfahrung und anerworbener, gelehrter Abstraktion.

\subsection{Das Buch «De signorum medicorum doctrina» von Emmanuel Stupanus (Basel 1649)}

Emmanuel Stupanus (1587-1664) war Professor in Basel und hat in einer acht Punkte enthaltenden Disputation ${ }^{24}$ rund 70 Jahre nach Aubert seine Ansicht zur medizinischen Zeichenlehre vorgetragen. Die Autoritäten, die er dabei zitiert, sind das hippokratische «Prognostikon», galenische Schriften, vor allem aber «De locis affectis», sowie das Kapitel «De symptomatibus et signis» aus Fernels oben vorgestellter «Pathologia». Seine acht Abschnitte beziehen sich auf:

\footnotetext{
Allgemeine Zeichen der Krankheit und Gesundheit.

Die pathognomonischen Zeichen, auch diagnostische genannt. Es sind dies die Zeichen des Ortes und der «retenta», der zurückgehaltenen Körperausscheidungen (materia peccans).

Die aus der Krankheitsursache erschlossenen Zeichen, wobei die Krankheitsursache als bekannt vorausgesetzt ist.
} 
Die prognostischen Zeichen.

Die Zeichen des Geschlechts und Lebensalters.

Die Pulszeichen, die auf die Lebenskraft hinweisen.

Die Zeichen aus dem Urin, die auf Fieber, Krämpfe usw. deuten.

Die Zeichen der Krankheitskrise.

Wiederum ist das Zeicheninventar recht vollständig, doch ist die Logik etwas verwirrlich. Es werden da einerseits Zeichen, die eindeutig «sensibus suis», wie es Aubert sagte, als gleichwertig neben solche gestellt, die aus als wohlbekannt vorausgesetzten Tatbeständen erschlossen werden müssen.

Die als gültig erachtete Lehrmeinung steht demnach als Zeichen gleichrangig neben der beobachtbaren Tatsache. Diese Vermischung zwischen theoretischer Lehrmeinung und praktischer Beobachtung ist insofern bedauerlich, als sie in der Semiotik Schule machte und den Konsens verzögerte, was denn eigentlich als Zeichen beobachtet werden müsse. Zeichenlesen und Zeichendeuten ist nicht klar gesondert, obschon Stupanus den prinzipiellen Unterschied sah und vom Zeichenlesen zur Zeichendeutung auf die Pathologie hin schreiten wollte.

\subsection{Das Buch «Physiologische und pathologische Zeichenlehre» von C.G.Gruner (Jena 1801)}

Christian Gottfried Gruner (1744-1815) war Arzt im Weimar der Goethezeit. Gruner nennt zuerst:

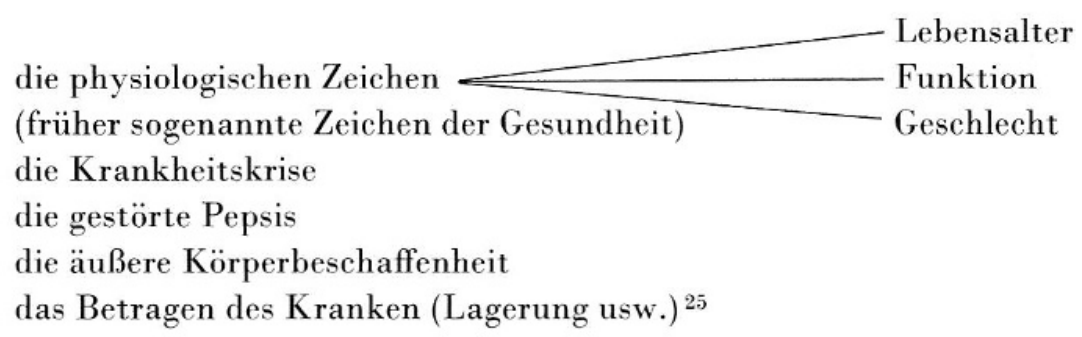

Diese äußerst knappe Zusammenfassung der Grunerschen Zeichenlehre, die übrigens eine zeitgemäß aufklärerische Vorrede enthält, erscheint auf den ersten Blick galenisch/altmodisch. Doch um Gruner Gerechtigkeit widerfahren zu lassen, muß gesagt werden, daß er im einzelnen auf der Höhe des zeitgenössischen Wissens stand. Was ihn von den vorangegangenen Semiotikern unterscheidet, ist die konsequente Systematisierung der Zeichen aus den Funktionen, was eine beträchtliche Objektivierung des Zeichens an sich zur Folge hatte. 
Wenn man sich nun fragt, worauf die Zeichen bei Gruner deuten, muß man antworten, daß er diese Frage ziemlich offen läßt. Natürlich deuten sie auf Krankheit im allgemeinsten Sinn, aber sie deuten nicht mehr ausdrücklich auf eine noch so modernisierte galenische Pathologie.

\subsection{Bemerkungen über weitere Lehrbücher der Semiotik des 18. und 19. Jahrhunderts}

Die Lehrbücher von Stupanus und Gruner können in ihrer Einteilung der Zeichen als ziemlich typisch gelten. Wir finden Ähnliches noch bis $1850^{26}$. Ohne den Anspruch auf Vollständigkeit zu erheben, finden wir in den Bibliographien ${ }^{27}$ von 1550 bis 1800 gut und gern 70 bis 80 verschiedene Autoren verzeichnet, die über medizinische Semiotik publizierten. Es darf daraus geschlossen werden, daß die Zeichenlehre damals mindestens kein vernachlässigtes Gebiet war. Doch gewinnt man den Eindruck, daß sie auf den von Stupanus u. a. vorgezeichneten Bahnen weiterging.

Die Titel der verschiedenen «Semiotiken» verraten, daß sich bei dieser Wissenschaft ein gewisser Zwiespalt und Widerstreit abzeichnete, wie wir ihn schon zwischen Aubert und Stupanus gesehen haben. Bei den einen Autoren überwiegt, wie bei Aubert, die reine Zeichenlesung, die sich nicht viel um die Pathologie kümmerte. Bei den anderen herrschte, wie bei Stupanus, eine Vermischung der Zeichenlesung mit der auf die Pathologie weisenden Zeichendeutung, wobei die Pathologie noch lange ein - wenn auch modifizierter - Galenismus war.

Alle diese Ärzte hatten das Bestreben, das Zeichen zum Grundtatbestand der Medizin zu machen, und zwar in dem Sinne, wie etwa Newton die Schwerkraft zum Grundtatbestand der Physik erklärt hatte. Damit wollten sie die Medizin in den Rang einer exakten Wissenschaft erheben. Folgerichtig gingen sie auch daran, die Vielzahl der Zeichen aufzuzählen und zu ordnen. Wir fügen bei, daß der Begründer der Homöopathie, S. C. Hahnemann, eine ähnliche Absicht verfolgte, dessen Zeichenaufzählung beim einzelnen Patienten bis zu 1000 gehen konnte ${ }^{28}$. Doch ist dies nun schon beinahe als Perversion der Zeichenlehre zu betrachten.

Der Anspruch der Semiotiker, die Zeichenlehre zur Basis der Medizin zu machen, beruft sich auf Hippokrates, mutet aber dennoch mit seiner Analogie zur Physik des Barocks und der Aufklärungszeit modern an. Wenn man aber anschaut, welche Zeichen im einzelnen die sogenannten Grundtatbestände sein sollen, zeigt sich die Verhaftung besonders der deutschen 
Semiotiker in der Tradition der «Semeiotica»-Lehrbücher des 17. Jahrhunderts.

Während die ersten Semiotikbücher des 16. und 17.Jahrhunderts noch im französischen Sprachgebiet (inklusive Schweiz) erschienen, macht es den Anschein, daß diese Wissenschaft bald ein deutsches Prärogativ wurde. Allein im Gebiet Halle/Leipzig/Wittenberg/Jena/Weimar und Erfurt wurden seit 1650 etwa 30 Zeichenlehren publiziert. Einige wenige stammen aus England, Holland und Italien. In Paris und Lyon wurden hin und wieder welche verlegt; jedoch entstand in Frankreich zur Wende des 18./19.Jahrhunderts der große «Dictionnaire des Symptomes et Eléments de Semeiotique», andererseits aber auch das «Handbuch der Semiotik» von Kurt Sprengel (Halle 1801). Die Erklärung, weshalb Deutschland das Land der Semiotik wurde, müssen wir hier noch schuldig bleiben.

Es ist schließlich nochmals festzuhalten, daß die «Semeiotica» recht eigentlich Lehrgänge für angehende Ärzte wurden, gewissermaßen Kochbücher der medizinischen Kunst. Die Zeichenlehre behielt demnach den Status einer Hilfswissenschaft, den sie schon seit Galen innehatte, einer nützlichen Magd, die zwar jeder Arzt anstellen mußte, die aber der wissenschaftlichen Bearbeitung nicht für ganz würdig erachtet wurde. Mit Semiotik konnte man sich nur bei den Studenten Lorbeeren ernten.

\subsection{Die Systematiker: François Boissier de Sauvages und die Nosologen des 18. Jahrhunderts}

Wie wir gezeigt haben, emanzipierte sich das Zeichen dank den Bemühungen von Gruner und anderer deutscher Semiotiker gewissermaßen von der Pathologie.

Ein Zeichen, das auf nichts hindeutet, ist aber eine Kontradiktion. Dabei kann es nicht bleiben. Daß es damit auch nicht sein Bewenden hatte, soll ein kurzer zeitlicher Rückgriff auf die Nosologen des 18. Jahrhunderts bestätigen.

Als ob schon ein allgemeiner Konsens darüber bestanden hätte, was ein Zeichen wäre, gab François Boissier de Sauvages 1763 in Amsterdam seine «Nosologia methodica» heraus. Unter dem etwas eigentümlichen Anspruch, die Krankheiten nach der Manier von Thomas Sydenham als quasi botanische Spezies aufzufassen, gruppiert Boissier de Sauvages die De-factoKrankheitszeichen, die «signa», zu spezies-morphologischen Einheiten, die überdies noch verwandtschaftliche Beziehungen als Krankheitsklassen 
haben sollten. Seine Idee, Krankheiten wie botanische Einheiten zu klassieren, scheint uns heute verfehlt, aber seine «Nosologia» enthält trotzdem eine höchst beachtliche Zeichenlehre. Die Zeichen sind folgende:

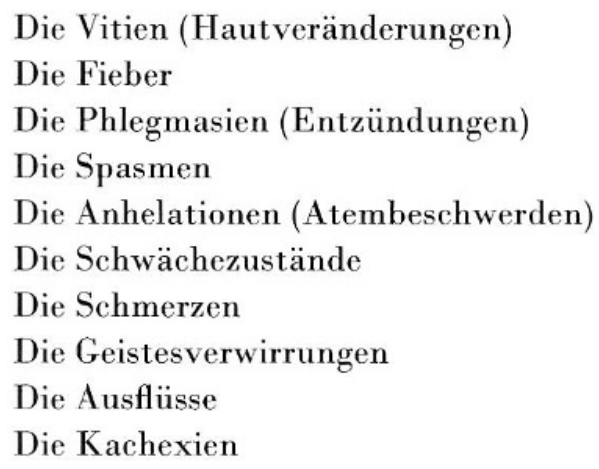

Die einzelnen Zeichengruppen sind unterteilt; seine «Nosologia» ist sehr ausführlich. Daß Boissier de Sauvages aus diesen 10 Krankheitserscheinungen ein Verwandtschaftssystem konstruierte, beschäftigt uns hier nicht. Vielmehr soll betrachtet werden, wozu sein Zeichensystem auch noch diente.

«Die Krankheiten sind das Zusammentreffen der auffallenden und wechselseitig verbundenen Symptome. Die mehr auf der Hand liegenden und gleichzeitg unwandelbaren Symptome führen den Zug (der Symptome) an und bestimmen den wesentlichen Charakter der Krankheit. Diese Symptome nennt man pathognomonisch. Die charakteristischen Zeichen sind dreierlei Art: in den Funktionen, den Ausscheidungen und den Qualitäten ...» ${ }^{29}$

Wie aus diesem Zitat hervorgeht, faßte Boissier de Sauvages die Zeichenkombination nicht als eine auf die Pathologie bezogene auf, sondern lediglich - wie wir es auch heute sagen - als ein Syndrom, d.h. Zusammenfallen der charakteristischen Zeichen. In einem ersten Schritt ging er analytisch vor, indem er die Zeichen aufzählte. Durch diese begriffliche Isolation wurde der zweite Schritt, die Synthese zum Syndrom, vorbereitet. Syndrome sind uns heute als Krankheitsbilder gegenwärtig. Man nennt Boissier de Sauvages und die anderen Nosologen wie Carl Linné, William Cullen usw. in der Literatur gemeinhin sterile Systematiker im Gegensatz zu den fruchtbaren pathologischen Anatomen. Wir wollen uns auf diese Diskussion nicht weiter einlassen, sondern festhalten, daß sie auch im positiven Sinne Systematiker genannt werden können, weil sie durch die Systematisierung der Zeichen die Klarheit der Semiotik vorangetrieben haben. Der Schritt zur Synthese von Syndromen war nun nicht mehr weit.

Zu Ende des 18. Jahrhunderts stellen wir zusammenfassend die generelle Emanzipation des Zeichens von fast jeglicher Pathologie fest. Während bei 
den deutschen Semiotikern beinahe ein Nihilismus der Zeichendeutung entstand, verfolgten die Nosologen in Frankreich und England das ambitiöse Projekt, ein Krankheitensystem, eben eine Nosologie, aufzustellen. Dieses Projekt war utopisch verfrüht, weil das pathologische Grunderfordernis der Bestimmung des Ortes der Krankheit noch weitgehend unerfüllt war.

\section{Medizinische Semiotik im modernen Sinn}

\subsection{Die Kliniker: René-Théophile-Hyacinthe Laennec und die pathologische Anatomie}

Wie bekannt ist, publizierte Giovanni Baptista Morgagni 1761 sein Buch «De sedibus morborum ...», das inhaltlich den beinahe gleichen Titel trägt wie Galens «De locis affectis». Morgagnis «Vom Sitz der Krankheiten» enthielt die bis dahin ausführlichste Beschreibung der pathologisch veränderten Anatomie. Das Licht wurde vor allem auf die inneren Krankheitszustände geworfen, die sich notwendigerweise in den zahlreichen äußeren Zeichen darstellen mußten.

Seitdem nach 1700 in den großen Städten zunehmend Krankenanstalten gegründet bzw. reorganisiert wurden, entstand eine neue Kategorie von Ärzten, die sich nicht mehr lediglich auf ihre sporadischen Beobachtungen am Kranken verlassen mußten. Man nennt diese Ärzte die Kliniker. Beispielshalber sei Herman Boerhaave, Gerhard van Swieten, Leopold Auenbrugger und eben Laennec genannt. Durch das Zusammentreffen einer numerischen Vielfalt von Krankheitsfällen konnten Vergleiche angestellt werden, die das Erkennen von Krankheitsbildern begünstigte. Durch die allmähliche Zunahme der Autopsien in diesen Krankenhäusern konnten diese Kliniker auch selbst die Brücke zwischen dem «klinischen» Zeichen und dem Ort der Krankheit im Inneren des Körpers schlagen.

Mit der Perkussion versuchte Auenbrugger schon am Kranken die inneren pathologisch/anatomischen Vorgänge $\mathrm{zu}$ «erspähen». Dieselbe Absicht, womöglich schon am Kranken das Körperinnere auf pathologisch/anatomische Veränderungen zu erkunden, verfolgte auch René-Théophile-Hyacinthe Laennec, als er 1816 das Stethoskop erdachte, damals eine unerhörte diagnostische Neuheit. Ist Laennecs erste Ausgabe seines Buches über das Stethoskop, das er «Ausculation Médiate» (1819) betitelte, noch 
vorwiegend mit den den Krankheitszeichen entsprechenden Geräuscherfahrungen befaßt, so ist die zweite Ausgabe des Buches (1826) völlig mit der Koordination von (Geräusch)-Zeichen und «locus affectus» bzw. «sedes morbi» beschäftigt.

«Je me contenterai donc de décrire les maladies des organes thoraciques, c'est à dire les cas pathologiques tranchés et distincts les uns des autres. J'exposerai les caractères auxquels on peut les reconnaître sur le vivant et sur le cadavre. J'indiquerai ensuite les méthodes de traitement...» ${ }^{30}$

Dieses Programm, das die charakteristischen Zeichen, die der Kranke aufweist, mit den Läsionen, die an der Leiche zu finden sind, koordinieren soll, hat Laennec in der zweiten Ausgabe seines oben erwähnten Buches mit großartiger Konsequenz durchgeführt. Das Werk ist bis heute ein Klassiker der Lungen- und Herzkrankheiten geblieben. Dergestalt das Krankheitszeichen mit dem Ort der Läsion im Körperinneren verbindend, schlägt Laennec die Brücke vom Zeichen zum Ort der Krankheit, wie das schon Galen vorgeschwebt hatte. Er gibt die gültigen Beschreibungen der Bronchitis, der Pneumonie, der Pleuritis, der Lungenembolie, der Lungengangrän, der Tuberkulose, der Herzhypertrophie sowie verschiedener Herzklappenfehler, die für den Arzt auch heute noch Namen von Krankheiten sind.

\subsection{Die praktische Semiotik der Kliniker des 19. Jahrhunderts}

Die Laennecsche Methode der Koordination des klinischen Zeichens mit dem Ort der Krankheit wurde zum Vorbild. Von ungefähr 1800 an und durch das ganze 19. Jahrhundert hindurchziehend, finden wir Krankheiten und Syndrome beschrieben, an denen sich der heutige Arzt noch orientiert. Sie sind der Kürze halber oftmals mit den Eigennamen ihrer Entdecker bezeichnet. Wir erwähnen Parkinson (1817), Hodgkin (1832), Basedow (1840), Addison (1849), Brown-Séquard (1850), Mênière (1861), Paget (1877), Perthes (1910).

Diese zufällige Liste ist natürlich nicht vollständig. Doch fällt auf, daß sie vorwiegend französische und angelsächsische Namen aufweist. Das mag einerseits einen Rückschluß auf die Anhänglichkeit dieser Nationen an ihre großen Kliniker erlauben, vielleicht aber auch bedeuten, daß sich die Ärzte in diesen Ländern vermehrt der Methode der syntaktischen Semiotik bedient haben. Die syntaktische Beschreibung von Krankheitsbildern hat sich seit dem 1. Weltkrieg sehr verfeinert und verästelt; es sei denn, es treten 
sogenannte neue, bis dahin nicht als solche identifizierte Krankheiten an den Tag, wie z. B. der «Legionaire's Disease» und AIDS.

Wir stellen demnach im 19. Jahrhundert einen Höhepunkt der Zeichenlesung fest, wie er seit den Zeiten der hippokratischen Medizin nicht stattgefunden hatte. Die Bücher, die sich «Semeiotica» nennen, verschwinden allerdings fast ganz oder werden zu Leitfäden für Studenten der ersten Semester. Für höhere Wissensgrade und ausgebildete Ärzte wird Semiotik heute unter Titeln wie «On Symptoms and Signs» ${ }^{31}$ oder «Differentialdiagnose» ${ }^{32}$ gehandelt.

\subsection{Pathologische Anatomie und Ätiologie}

Hat durch die Isolation des Zeichens an sich während des 18. Jahrhunderts die syntaktische Semiotik im 19.Jahrhundert eine rasche Ausbildung erfahren, so finden wir einen ähnlichen Aufschwung auch auf den Gebieten der pathologischen Anatomie und Ätiologie.

Wenn auch anekdotisch, so ist doch Rudolf Virchows Frage an seine Studenten: «Ubi morbus?» sehr charakteristisch für die Wichtigkeit, die nun dem «locus affectus», d. h. dem anatomischen Substrat der Krankheit, die so viele Zeichen machen konnte, zugemessen wurde. Diese Bemerkung genüge, um auf die Geschichte der pathologischen Anatomie hinzuweisen.

Nun, da man wußte, daß bestimmte Zeichengruppierungen, d.h. Syndrome oder Krankheiten, einen umschriebenen Ort bedeuteten; nun, da man die Zeichen auf die pathologische Anatomie bezog, war es auch klar geworden, wofür man eine Ätiologie zu suchen hatte.

Zwar spielte die Suche nach übernatürlichen und später natürlichen Ursachen seit den ältesten Zeiten der Medizin eine wichtige Rolle. Besonders seit der Renaissance waren die medizinischen Abhandlungen wiederum überfüllt mit vermutlichen natürlichen Krankheitsursachen. Man sah in den Konstellationen, den klimatischen Bedingungen, im Essen, im Laster, im Lebenswandel Ursachen, mit denen wir uns zum Teil auch heute noch auseinanderzusetzen haben.

Während es also nie an Ideen gemangelt hat, wo man Ursachen suchen könnte, war bis zum Erreichen einer gewissen Reife der Semiotik im oben beschriebenen Sinne, d.h. bis ungefähr um 1800, unklar, wofür man überhaupt Ursachen suchen sollte. Es erstaunt daher keineswegs, daß im 19. Jahrhundert Wissenschaften wie die Chemie und die Mikrobiologie der klininischen Semiotik wesentliche Hinweise bringen konnten. So ist denn die 
Arbeit der Bakteriologen eine der großen Leistungen des 19. Jahrhunderts geworden, indem die Ätiologie so zahlreicher Infektionskrankheiten (wie der «Fieber») auf festere Füße zu stehen kam. Ebenso wurden zahlreiche der gesuchten «loci affecti» bekannt.

\subsection{Zusammenfassung}

Die Semiotik bleibt nach wie vor hochaktuell für die klinischen und praktischen Ärzte. Ihre tägliche Arbeit besteht in der korrekten Lesung der Zeichen, d.h. dem, was seit altersher Stellung einer Diagnose heißt. Wir haben dieses Wort bis anhin absichtlich zu vermeiden gesucht, weil es die Konnotation des Wissens hat. Wie wir dargestellt haben, war dieses Wissen in der Zeit vor einer ausgebildeten Semiotik höchst prekär. Aber natürlich bedeutet die Geschichte der Semiotik nichts anderes als die Darstellung des historischen Weges, den die Medizin beschritt, um es dem Arzt zu ermöglichen, überhaupt eine korrekte Diagnose zu stellen.

Wenn wir nun den Weg der medizinischen Semiotik noch einmal synoptisch überblicken, konstatieren wir, daß es nicht so sehr die begriffliche Einsicht in die Semiose war, die fehlte. Die Methode des Zeichenlesens wurde durchaus erkannt. Der Konsens aber, was als Zeichen betrachtet werden mu $\beta$, bildete sich erst allmählich heraus. Der Grund der Blickverdunkelung bestand darin, daß bis ins 18. Jahrhundert das Wissen, was Krankheit sei, als bekannt vorausgesetzt wurde. Weil die aprioristische Definition der Krankheit eine so allgemeine war (Dyskrasie, Funktionsstörung, «locus affectus»), meinten die Ärzte zu wissen, daß die Zeichen auf die vorgegebene Krankheit deuteten, ja deuten mußten.

Wenn man von den deutschen naturphilosophischen Ärzten absieht, geben sich die Ärzte seit 1800 nicht mehr primär mit der Frage ab, was Krankheit sei. Krankheit ist Krankheitsbild geworden, d.h. Einheit, deren selbständige Zeichen es korrekt zu lesen gilt. Das Krankheitsbild als diagnostische Einheit rechtfertigt, vom Namen der Krankheit zu reden. Dabei sind die Forschungsbedingungen im allgemeinen auch heute nicht prinzipiell anders geworden, beruht doch die Zeichenlesung nach wie vor auf der Wahrnehmung des einzelnen Beobachters. 


\section{Anmerkungen}

1 Th. Seboek Theorie und Geschichte der Semiotik, 1976; deutsch: Hamburg 1979.

2 A. und M.Stettler, Zur Semiotik ärztlichen Handelns, in: Schweizerischer Rundschau für Medizin «Praxis» 28, 1986, 857-861.

3 Ch. Morris, Grundlagen der Zeichentheorie, 1938; deutsch: Frankfurt/M 1979.

4 Corpus Hippocraticum; «Prognostikon» in: Ed. W. Capelle, Zürich 1955.

5 op.cit.sub no.4: Liste, hergestellt aus den Kapiteln des «Prognostikons», in: Ed. W. Capelle, Zürich 1955.

6 op.cit.sub. no.4: «Epidemien» erster Fall, in: Ed. W. Capelle, Zürich 1955.

7 op.cit.sub no.4: «Von den Frauenkrankheiten», Einleitung, in: Ed. W. Capelle, Zürich 1955.

8 F. Kudlien, zit. in W. Eich, sub no. 9.

9 W. Eich, Medizinische Semiotik zwischen 1750 und 1850, Diss. med. Freiburg/B 1980 (im Manuskript).

10 Aretaios von Kappadokien, zit. in: R.H. Major, Classic Description of Disease, Springfield/Ill. 1955, S.136, bzw. Corpus medicorum graecorum Bd.II, Aretaios, Buch IV, Kap. 13.

11 Rhazes, zit. in R.H. Major, op. cit.sub no. 10, S. $196 \mathrm{ff}$.

12 Pestbeschreiber: zit. in R.H. Major, op. cit.sub no. 10; ferner G. Sticker, Abhandlungen zur Seuchengeschichte, Gießen 1908, und J.-N.Biraben, Les hommes et la peste..., Paris 1975.

13 Th.Sydenham, Dissertatio epistolaris ... de affectione hysterica, London 1685, zit. in opera omnia, London 1844.

14 Th.Willis, Oxford Casebook; Ed.: K.Dewhurst, Oxford 1981; F. Platter, Observationes; Ed.: H. Buess, Bern 1963.

15 Galen von Pergamon, Ed.: Kühn, 19, 351.

16 Galen von Pergamon, Ed.: Kühn, 14, 690 (Introductio seu... Kap. 7).

17 Avicenna, Canon medicinae, Buch I, Fen 1+2, Ed.: Juntas, Venedig 1595. (Die Randbemerkungen dieser Ausgabe weisen auf die Galenstellen, auf welche der «Canon» Bezug nimmt. Die Tradition ist gesichert.)

18 Avicenna, op. cit.sub no. 17, Buch I, Fen 2, Doct.3.

19 J. Fernel, Pathologia, in: Medicina, Paris 1554, Buch II, Kap. 1.

20 ibidem.

21 Dictionnaire des Sciences Médicales, Paris 1812-1822, Stichwort: symptomatologie.

22 A. Benivieni, De abditis... morborum causis, Venedig 1507.

23 J.Aubert, Semeiotica sive ratio diagnoscendarum sedium male affectarum et affectuum praeter naturam, s.l. 1632.

24 E.Stupanus, Signorum medicorum doctrinam, adnexa sphygmice, uromantia et crisium theoria, Basel 1649.

25 C.G.Gruner, Physiologische und pathologische Zeichenlehre, Jena 1801.

26 op.cit.sub no.9. (Neben dem Werk von C.G.Gruner werden noch die Lehrbücher der Semiotik von E.A.Nicolai, J.D.Grau, F.G.Danz, J.C.A. Heinroth und K.C. Wolfart untersucht.) 
27 Bibliographien über Semiotikbücher, in: C.G.Gruner, op.cit.sub no.25; Dict.d. Sciences Médicales, op.cit.sub no.21; Boerhave/Haller, Methodus studii medici ..., Amsterdam 1751.

28 H.J.Schwanitz, Homöopathie und Brownianismus, Stuttgart/New York 1983.

29 F.Boissier de Sauvages, Nosologia Methodica, Amsterdam 1763, Prolegomena.

30 R.T.H.Laennec, Traité de l'auscultation médiate et des maladies du poumon et du cœur, Paris 1826, S. 133.

31 C. M. McBride, Signs and Symptoms, Philadelphia 1964, 4. Aufl.

32 R.Hegglin $/$ W. Siegenthaler, Differentialdiagnose innerer Krankheiten, Stuttgart 1952 bzw. 1980 (14. Aufl.).

\section{Summary}

The new science of semiotics, dating from the 19th century (de Saussure [1857-1913]), claims Hippocrates among its founding ancestors. In this article, an outline of the basics of medical semiotics is given, i.e. the art of reading and interpreting signs. There follows a survey of Hippocratic and Galenic sign-reading in medecine. In the Renaissance, medical sign reading became a science on its own, viz. treatises with the title "Semeiotica» were numerously published. The art of medical semiotics, sign-reading, experienced a peak with Laennec and his contemporaries, when sign reading established entities of disease, which are valuable up to this day.

Frau Dr. med. Antoinette Stettler

Kirchenfeldstraße 45

CH-3006 Bern 